Linguistique, littérature, didactique

151-152| 2011

Anthropologies de la littérature

\title{
Littératures et formes de vie
}

\section{Florent Coste}

\section{(2) OpenEdition \\ Journals}

Édition électronique

URL : http://journals.openedition.org/pratiques/1788

DOI : 10.4000/pratiques. 1788

ISSN : 2425-2042

\section{Éditeur}

Centre de recherche sur les médiations (CREM)

\section{Édition imprimée}

Date de publication : 15 décembre 2011

Pagination : 73-88

\section{Référence électronique}

Florent Coste, «Littératures et formes de vie », Pratiques [En ligne], 151-152 | 2011, mis en ligne le 16 juin 2014, consulté le 01 mai 2019. URL : http://journals.openedition.org/pratiques/1788 ; DOI : 10.4000/pratiques. 1788

(C) Tous droits réservés 


\section{Littératures et formes de vie}

\section{Florent Coste}

Université de Bourgogne

\section{Anthropologie et littérature : les raisons d'un rendez-vous manqué}

Pour nécessaire qu'il nous semble, le rapprochement entre poétique des textes littéraires et concepts des sciences sociales continue d'apparaître incongru et laborieux. Le partage entre analyse interne (supposée entrer dans la chair profonde des textes) et analyse externe (supposée survoler l'environnement de l'œuvre) reste en effet prégnant et continue de cliver les disciplines comme l'histoire littéraire et l'histoire de la lecture, la sociocritique et la sociologie de la littérature. Ce faible taux de pénétration des sciences sociales dans les études littéraires n'en demeure pas moins à expliquer. C'est probablement que les principaux outils d'analyse spécifiquement littéraires prennent naissance au XIX ${ }^{\mathrm{e}}$ siècle dans les moments historiques d'autonomisation d'un champ littéraire qui se donne pour caractéristique constitutive de masquer ses propres déterminations sociales et historiques (Bourdieu, 1998 ; J.-P. Martin, 2010). On a sans mal dressé une généalogie du structuralisme et du formalisme remontant jusqu'à la conception de la littérature que le romantisme d'Iéna (Lacoue-Labarthe et Nancy, 1978) a longtemps colportée en prétendant arracher cet autre langage que serait la littérature de l'ordinaire de la vie pour la sublimer dans une théologie idéaliste tendant à sacraliser l'art (Schaeffer, 1992 ; Bessière, 2001 ; Maingueneau, 2006 ; Cometti, 2000, 2009).

Cette conception a trouvé un relais fort dans le Contre Sainte-Beuve de Proust qui radicalise la mythologie de l'esthétique romantique. La distinction, qui y est défendue entre d'une part le moi social de la conversation profane et de la mondanité vulgaire et d'autre part le moi créateur du retranchement et de la vie artiste, a pour conséquence direct de minimiser l'environnement biographico-institutionnel et de prôner une déconnexion entre la sphère esthétique et la sphère sociale. Sur ces bases, Proust considère qu'on se donne accès à un niveau de lecture adéquat de l'œuvre, parce que profond, global et dépris des considérations extérieures qui rappellent le créateur à sa finitude et atomisent la lecture en des détails insignifiants. C'est là l'une des racines du partage entre analyse interne (à même de ressaisir la totalité organique de l'œuvre) et analyse externe (soucieuse de futiles contingences) sur lequel les départements de Lettres modernes, via la Nouvelle critique, ont légitimé leur existence (Maingueneau, 2006), négligeant de prodiguer une formation décente dans les différentes sciences sociales. 
De sorte qu'une analyse de type organiciste, même si elle s'applique à la littérature $\mathrm{du} \mathrm{XIX}^{\mathrm{e}}$ et du XX $\mathrm{XX}^{\mathrm{e}}$ siècle qui en a posé les jalons, se rend coupable du même manque de réflexivité que commettrait un observateur qui se contenterait de décrire une société en tenant pour argent comptant le sens commun de cette dernière. On ne lui accorderait guère le bénéfice de la scientificité. Les outils hérités de la Nouvelle Critique, en plus de s'être banalisés dans le bagage théorique littéraire, charrient avec eux une tendance à un mépris axiologique, dont l'ultime conséquence serait de dévaluer dans un historicisme évolutionniste et essentialiste toute littérature qui ne tendrait pas vers les circonvolutions réflexives d'un Proust ou d'un Mallarmé - avènements de la vraie Littérature $^{(1)}$. Une sécularisation de la littérature s'impose, pour en finir avec sa sacralité romantique et son statut d'exception (Bessière, 2001), tout autant qu'une décolonisation des prismes de la modernité qui n'ont cessé d'être plaqués, en amont et en aval du romantisme. C'est en ce sens qu'il y aurait à défendre une anthropologie de la littérature.

\section{Qu'entend-on par « anthropologie de la littérature»?}

Pour qu'une telle entreprise puisse cependant être menée à bien, il est nécessaire de clarifier les raisons qui nous autorisent à faire des usages anthropologiques de la littérature qui ne soient pas seulement documentaires. Les sciences humaines actuelles importent ce terme à tout le moins séduisant d'anthropologie pour divers motifs ${ }^{(2)}$.

(1) On convoque l'anthropologie en raison de l'objet d'étude (comme la parenté, les techniques du corps ou le don; ou des ethnies comme les Jivaros, les Nuer, les Dogons...) : on construit, circonscrit et isole alors cet objet plus ou moins lointain ou profond sous la forme d'une totalité différente, close et représentative (c'est en partie d'un tel usage de l'anthropologie que font la microstoria ou l'anthropologie historique).

(2) On prétend à l'anthropologie quant à la méthode employée et à l'écriture ethnographique mise en œuvre. Pensons à B. Latour et à la Vie de Laboratoire (1988) : 1'ethnographe s'immerge dans son terrain et tente de s'y faire oublier pour observer les acteurs, au gré d'une suspension de tous les jugements de valeur.

(3) Enfin on s'autorise à parler d'anthropologie, au regard de résultats heuristiques spécifiques ; l'anthropologie se définirait par la mise en œuvre d'une posture compréhensive, par un principe de charité (Quine, 1977 ; Davidson, 1993) accordant du sens à ce qui semble le plus étrange et le plus irrationnel (Gell, 2009, p. 12), par un aller-retour critique entre catégories savantes et catégories indigènes (Olivier de Sardan, 1998) et par le refus de tout constructivisme à sens unique (Viveiros de Castro, 2009, p. 5 et suiv.).

Culminant ainsi dans la lutte de la réflexivité contre un ethnocentrisme initial, l'anthropologie est érigée en un modèle de la connaissance interculturelle probant, reposant sur un postulat universaliste de comparabilité et de traductibilité des cultures, et capable de franchir les cloisons disciplinaires autant que celles que dresse un monde pourtant globalisé. L'anthropologie constitue à cet égard autant un corpus de textes, d'outils, de concepts, qu'un ethos scientifique et méthodologique dont l'attractivité repose sur plusieurs de ses caractéristiques : l'explication par la culture, le contextualisme, la focalisation sur l'altérité, l'interdisciplinarité et l'auto-critique (Foster, 2008, pp. 116-117).

(1) Voir les critiques méta-théoriques constructives de Christophe Hanna (2010, pp. 36-39) contre les poétiques historicistes, qui par un conservatisme intrinsèque ne parviennent pas à penser la radicale nouveauté.

(2) Hal Foster jette un regard plutôt blasé sur la tendance de l'anthropologie à s'infiltrer dans l'histoire de l'art et les visual studies : «L'anthropologie demeure le discours de compromis par excellence » (2008, pp. 118). 
Puisqu'il s'agit de corriger notre regard sur la littérature, coupable d'un ethnocentrisme romantique qui se légitime et s'autonomise en se dotant de corpus canoniques et d'outils descriptifs qui se co-construisent de manière tautologique, nous souhaiterions plaider en faveur d'une anthropologie de la littérature forte de ressources thérapeutiques : sa vertu serait de nous renvoyer une image de notre rapport à la littérature où nous ne nous reconnaîtrions pas. En refusant à la littérature quelque statut réservé que ce soit, en doutant de notre capacité à discriminer ce qui en relève et ce qui n'en relève pas, en soumettant les instruments de l'analyse littéraire, possiblement inadéquats, à un certain soupçon, en se plaçant au ras des pratiques d'écriture littéraire dans leur fonctionnement même et sans préjuger, en vertu d'un principe de symétrie et d'impartialité ${ }^{(3)}$, de leur qualité, de leur réussite et de leur conformité à un canon, on doit se donner les moyens d'en décrire leur inscription et leur efficacité dans le monde.

\section{Anthropologie sociale vs anthropologie pragmatique}

Mais l'anthropologie n'est pas un territoire unifié, malgré l'ombre portée du structuralisme lévi-straussien en France. Il est nécessaire ici de se positionner par rapport à deux traditions anthropologiques. Schématiquement on peut faire le distinguo entre d'une part l'anthropologie structurale et sociale et d'autre part l'anthropologie pragmatique. L'anthropologie sociale constitue un modèle dominant qui conçoit la recherche anthropologique comme une extrapolation de données particulières par comparaison: l'observation (ethnographique et locale) de terrain doit alimenter d'abord un travail (ethnologique) d'identification culturelle d'une altérité, puis de rangement et de classification (anthropologique, naturaliste et globale) de la diversité humaine au moyen de l'outil de structure. Si l'on suit les arguments décisifs de Jean Bazin (2008), l'anthropologue structural se donne pour mission d'exhumer la couche profonde de la culture pour expliquer les gestes de tel ou tel membre d'une ethnie donnée. Son raisonnement prend la forme suivante : le Bambara agit de la sorte, parce que sa bambaraité le lui dicte. De sorte qu'on construit de manière tautologique une identité ethnologique censée expliquer ce que l'on vient de décrire : «c'est expliquer la manière dont ils agissent par la manière dont ils agissent » (Descombes, in Bazin, 2008, pp. 20-21). La postulation de structures qui transcenderaient les acteurs, les objets et les pratiques ne constitue finalement qu'un sophisme circulaire. À travers l'explication par « la culture », on subordonne les pratiques à des essences disjointes et exotiques et on réifie les acteurs dans des identités forcloses et éthérées censées tout expliquer.

Ces objections frappent autant le structuralisme que l'herméneutique qui dérivent dans les mêmes régressions infinies de la règle, de la structure ou du noyau de sens. De même les notions bien connues de l'histoire de l'art, comme le Kunstwollen, la « forme symbolique », «l'œil d'une période», le «style cognitif» sont autant de fétiches théoriques (Foster, 2008, pp. 110-114), qui n'échappent pas à ces critiques. Ces dernières sont instructives plus largement car elles rappellent que rien ne nous autorise à statuer sur ce que sont les individus que nous observons plus ou moins directement. Il ne relève tout au plus de notre juridiction que de décrire ce qu'ils font ou ont fait. Voilà pourquoi, si l'explication par « la culture » vacille, s'en remettre à une anthropologie «pragmati-

(3) Nous renvoyons à l'un des piliers sur lequel la sociologie et l'histoire des sciences depuis D. Bloor et son strong program s'est érigé. En vue de contrer la téléologie de l'épistémologie, il repose sur plusieurs principes : à un principe de symétrie recommandé pour analyser équitablement des théories divergentes s'en ajoutent d'autres qu'on oublie plus facilement : un principe d'impartialité à l'égard des croyances jugées rationnelles ou non, un principe attentif à la pluralité des causalités à l'œuvre dans l'élaboration des connaissances et enfin un principe de réflexivité concernant l'utilisation des connaissances scientifiques dans l'analyse sociologique. 
que », plus attentive aux actions et aux pratiques elles-mêmes riches d'un sens à décrire qu'à l'esprit censé les gouverner ${ }^{(4)}$. En déplaçant le curseur de la question de l'identité à celle de l'action, une anthropologie de la littérature serait en ce sens plus intéressée au langage comme acte immanent que comme mode d'accès à une réalité secrète qui le régirait en amont ou en arrière-plan. En adaptant la formule d'Alfred Gell (2009, p. 33), une théorie anthropologique de la littérature est une théorie des relations sociales axée autour et tissée par des œuvres littéraires.

Dès lors, une voie d'approche anthropologique des objets d'art qui soit satisfaisante serait, loin de considérer les significations qui leur sont attachées ou les critères de beauté qu'ils devraient épouser, de les envisager comme des agents issus d'initiatives sociales et visant un effet certain sur le monde et spécifique au contexte auquel ils sont attachés ${ }^{(5)}$. L'un des bénéfices pour les études littéraires serait de ne jamais disqualifier la littérature dans la hiérarchie des sources historiques, sociologiques ou anthropologiques, au prétexte qu'elle imposerait un accès indirect ou un filtre interprétatif au réel (Kalifa, 2009, p. 93). Car ce serait précisément méconnaître, que tout texte - et l'art en général - est un objet digne d'attention, parce que fonctionnant comme un principe actif d'investissement et d'exploration du monde, qui au même titre que la science élaborent autant d'organes améliorant notre prise sur lui (Morizot, 2008). S'il importe de dépister les encodages, les dialogismes et les hybridations du contexte dans le texte, la contrepartie est de penser aussi l'objet social qu'est le texte littéraire comme doté d'un pouvoir ou d'un effet sur le contexte dans lequel il s'inscrit en tant que « système symbolique actif » (Hanna, 2002).

La question de la représentation littéraire s'en trouverait ainsi profondément rediscutée en ses termes : la littérature-miroir muterait en littérature modélisatrice ou formalisatrice de nos activités ordinaires ; dans le reflet qu'elle livre après coup de l'histoire, elle l'infléchirait du poids de ses mots ; la mimésis littéraire concevant le texte comme un réceptacle transparent captant des représentations flottantes s'effacerait au profit d'une présentification d'un texte-objet ${ }^{(6)}$, de processus, de performances qui ne cesseraient de peser dans le jeu social et d'y faire événement, en ouvrant des chemins d'accès à des voix et des causes, en faisant advenir dans l'espace public des êtres et des problèmes à représenter (au sens politique du terme). Une anthropologie pragmatique de la littérature privilégie l'action littéraire dans ses modalités concrètes, c'est-à-dire l'effectivité pratique, morale et cognitive de langages littéraires considérés dans leurs aspects opératoires. Elle appréhende l'œuvre littéraire moins comme un opus que comme un modus operandi déterminé autant que déterminant des relations sociales.

S'il y a en apparence quelque scandale à rabattre la littérature du côté des simples outils, on ne peut d'une part oublier les raffinements possiblement infinis de nos outils à travers les usages combinés et rusés que nous en faisons, d'autre part ni perdre de vue que la littérature, et l'art en général, a cette capacité d'élargir le champ de nos usages du

(4) Cette opposition entre anthropologie sociale et anthropologie pragmatique recoupe en grande partie le partage fait par Marshall Sahlins (1976) entre une anthropologie intéressée à la logique symbolique et reposant sur une conception de la société comme système d'échanges et une anthropologie tournée davantage vers la culture matérielle et la raison pratique. Clivage pour ainsi dire entre la textualité d'une part et la référentialité d'autre part (Foster, 2009, p. 117).

(5) Voir à ce propos les remarques d'un éminent représentant de l'anthropologie structurale, Philippe Descola, qui congédie une telle approche dans son cours au collège de France 2005-2006, " Modalités de la figuration ». [URL en ligne] : http://www.college-de-france.fr/media/anthrop/UPL35669_descola_cours0506.pdf (consulté le 19 août 2010)

(6) Sur la notion de présentification, voir Vernant (1996) : «La présentification est l'action ou l'opération par laquelle une entité appartenant au monde invisible devient présente dans le monde visible des humains "; on peut considérer que le texte littéraire, sans faire advenir des entités invisibles, intervient par les surcroîts de visibilité qu'il peut accorder ça et là ; sur le texte-objet, voir Chastang (2008). 
monde et d'accroître les prises que nous avons sur lui (Cometti, 2000 ; Dewey, 2010, p. 42). Par là on esquive un écueil théorique : réduire le langage à une source donnant sur une réalité qui lui serait extérieure, plus profonde et plus essentielle. Pour cela, nous avons besoin de nous équiper d'une conception du langage ordinaire, qui englobe, sans les exclure ni les marginaliser, les langages littéraires et qui le pense comme un mode d'action ouvert sur, et immergé dans l'immanence du réel.

Plusieurs, singulièrement des philosophes, ont convergé vers une telle pensée de la littérature, qui serait, sur fond d'un instrumentalisme, attentive à son impact cognitif, moral et pratique dans la vie quotidienne. Pensons au méliorisme de Stanley Cavell (1991, 1996), au progressisme de Martha Nussbaum (2010), à la philosophie morale de Cora Diamond, à la « connaissance» de Bouveresse $(2008)^{(7)}$. Sans que l'on puisse toutefois soutenir l'existence d'un courant qui les unirait, il n'est pourtant guère difficile de trouver un substrat commun à ces pensées pragmatiques de la littérature dans la théorie anthropologique du langage de Wittgenstein, qui constitue du reste l'une des sources de la critique de l'anthropologie sociale par Jean Bazin, évoquée plus haut ${ }^{(8)}$.

\section{Langage ordinaire, jeux de langage et formes de vie}

Une science sociale, quelle qu'elle soit, se confronte à des pratiques régulières, qu'elle veut décrire en tant que telles, et au besoin comprendre et expliquer. La question est de savoir s'il faut pour cela postuler une règle qui régisse ces conduites ou que les acteurs convoquent avant d'agir et ultimement si l'on peut y garantir un accès. Autrement dit de savoir si ces pratiques régulières sont des applications placées sous la dépendance logique et ontologique d'une règle ou si elles ne sont que l'usage de règles qui lui sont incorporées. Comme n'importe quel autre chercheur en sciences sociales, le chercheur en littérature n'y échappe pas. En dépit d'un pluralisme herméneutique largement admis, il endosse généralement la tâche de trouver la règle qui régit le texte (qu'on l'appelle la clé de lecture, l'interprétation adéquate ou la signification exacte de textes) pour esquiver les écueils d'un relativisme naïf des interprétations. Il se doit de répondre à la question suivante : faut-il en somme subsumer les pratiques observables sous des entités mentales, idéales, théoriques... et accorder un primat de la théorie sur la pratique (Bloor, 2001) ? Rien n'est en réalité moins sûr.

On peinerait en effet à interpréter le sens de la règle pour l'appliquer ensuite, emporté que l'on serait dans une dérive interminable d'interprétations d'interprétations de la règle. C'est en usant de la règle qu'on apprend sa signification. On ne peut dès lors déterminer la signification d'une règle, sans en référer aux pratiques auxquelles elle appartient. Une règle n'est compréhensible qu'inscrite dans le contexte d'une pratique ${ }^{(9)}$. Il n'existe donc pas d'esprit, de règle, de lieu mental qui serait dépositaire de la juste interprétation d'une règle ou de la signification correcte d'un mot. Nous ne possédons pas de critères privés pour discriminer la bonne mise en œuvre d'une règle et le fait de s'y conformer sans la suivre. Les seuls que nous avons à disposition sont publics et contextuels, et ce sont par exemple ceux qui nous permettent de distinguer le Quichotte de Cervantès et celui de Pierre Ménard. Sur fond de cette réfutation des conceptions mentalistes et intellectualistes de la signification qui assignent à l'esprit un monopole sur la production du sens, Wittgenstein défend une conception pragmatique du langage, pour la-

(7) Pour une critique de ce progressisme normatif, voir Macé (2011), qui rappelle la lecture à sa pesanteur, à ses contingences et à ses ratés.

(8) On ne compte guère les références explicites ou tacites à Wittgenstein dans l'œuvre de Jean Bazin.

(9) «La signification d'un mot est son usage dans le langage» (Wittgenstein, 2004, §43); «Ce n'est que dans la pratique du langage qu'un mot peut avoir une signification» (1983, p. 344). 
quelle l'usage linguistique ne provient pas de quelque façon de voir ou d'une quelconque conception qui se logerait en son amont dans une forteresse inaccessible de l'esprit (Cometti, 2000, p. 37). De plus, si l'élucidation de la signification ne peut passer sans aporie par l'articulation entre esprit et langage, du fait de la nature collective et publique de ce dernier ${ }^{(10)}$, les pensées de la représentation (ou de l'adéquation) commettent l'erreur de demander au langage de qualifier le monde de l'extérieur. L'apprentissage de l'usage des mots consiste surtout à apprendre à maîtriser les prises que les mots nous donnent sur le réel ou les ouvertures pratiques qu'ils inaugurent. On défend par là une dimension instrumentale du langage qui en fait un outil donnant des moyens d'action dans un monde auquel il est coordonné et qui ne lui est jamais extérieur.

Avec la notion de « jeu de langage », Wittgenstein invite à considérer dans son immanence un ensemble complexe et réglé, « formé par le langage et les activités avec lesquelles il est entrelacé » $(2004, \S 7)$. Le jeu de langage associe des objets linguistiques à des formes d'action et des habitudes de comportement. Il ouvre sur des usages et des pratiques, sans lesquelles il demeure incompréhensible ${ }^{(11)}$. En rivant tout jeu de langage à une forme de vie, on envisage le langage dans des contextes diversifiés d'énonciation, d'utilisation et d'application. Comme le dit Descombes (2004, p. 12), « les mots s'emploient dans un contexte et on explique leur sens en les y replaçant : d'abord dans celui de la construction d'une phrase, ensuite dans ceux d'un jeu de langage pour l'emploi de cette phrase et d'une forme de vie pour la pratique du jeu de langage. » Jeux de langage et formes de vie sont ainsi les revers indissociables l'un de l'autre, de sorte que le langage ne saurait être hétérogène au monde, ni à l'esprit. La forme de vie n'est pas un arrière-plan exerçant son déterminisme sur la surface des actions. "La forme de vie n'est pas constituée de pratiques, mais une forme que donnent les pratiques à notre vie et à notre connaissance. » (Laugier, 2009, p. 230). Il faut placer l'accent ici, comme a pu le faire Stanley Cavell $(1991 ; 1996)$, sur la dimension biologique de la « forme de vie» : c'est un accord si bien innervé dans nos pratiques, qu'il s'est quasiment naturalisé et qu'il échappe à tout partage nature/culture. En accédant ainsi à «l'absorption réciproque du naturel et du normatif » (Laugier, 2009, p. 233), on touche précisément ici à une convention devenue nécessité, qui relève sans mal du domaine de l'anthropologique (Lara, 2005 ; Chauviré, 2004 ; Das, 1998 ; Salgues, 2008).

Cette solution d'origine wittgensteinienne, dont on sait qu'elle fournira un socle aux théories des actes de langage (d'Austin ou de Searle) et à la pragmatique, porte en elle une redistribution des rapports entre texte et contexte. Le texte n'est pas seulement incompréhensible sans une contextualisation a posteriori, le contexte ne vient pas seulement fournir un supplément d'informations (et d'âme) à la compréhension du texte. La poétique se doit de prendre en compte dans un même mouvement le jeu de langage littéraire et la forme de vie qui lui est associée : le texte littéraire est constitutivement, et au même titre que n'importe quel autre objet social, un opérateur contextuel et immergé dans nos pratiques. Ainsi les approches contextualistes de la littérature ne peuvent plus se contenter d'enrober le texte des données qu'elles glanent pour en épaissir la lecture. Le contexte qui doit nous intéresser ne se situe ni en amont ni en aval du texte. Il en est inséparable.

Jeux de langage et formes de vie peuvent à cet égard enrichir l'arsenal théorique d'une poétique de la littérature aux ambitions pragmatiques et anthropologiques assumées et nous prémunir de certains errements théoriques. En déniaisant nos façons - défaillantes et aporétiques - d'inscrire le langage dans le réel, Wittgenstein propose de nous guérir de maux philosophiques - et par extension selon nous de maux littéraires :

(10) La réfutation de tout langage privé est menée par Wittgenstein dans les Recherches Philosophiques (2004). Voir également Jacques Bouveresse, Le mythe de l'intériorité, Paris, Minuit, 1976.

(11) «Imaginer un langage veut dire imaginer une forme de vie. » (Wittgenstein, 2004, §19) 
1. Le régulisme (Cometti, 2010, p. 323 ; Brandom, 2009) : à celui qui pense qu'une règle contient en germe toutes ses applications et possède un pouvoir causal mécanique sur les actions, il convient de rappeler que dans une telle hypothèse on ne parviendrait pas à expliquer les changements de règles ici soustraites à l'histoire et qu'une règle ne garantit pas à elle seule sa juste application. La littérature en donne assez d'exemples : une règle, une convention, un code générique se subvertit, se détourne, se contourne, se retourne, se truque ou s'inverse par les irrégularités que peut introduire à tout moment la littérature. Le déterminisme implacable des règles mérite d'être minoré au profit d'une historicité et d'une créativité réaffirmées de l'agir littéraire (Joas, 1999).

2. De même le mentalisme et l'intellectualisme, qui rivent le langage à des états mentaux, pèchent de se débrancher du plan de nos conduites ordinaires. La critique littéraire est concernée au premier chef, quand elle s'égare dans une abstraction textualiste déconnectée des modes d'inscription concrets du texte dans le jeu social ou dans une «pensée textuaire» (Cerquiglini, 1989), tributaire du clivage ruineux que l'imprimerie a instauré entre l'élaboration par l'auteur du discours et la production matérielle du livre par le libraire ou l'éditeur. Par delà l'opposition coûteuse entre matérialité du livre (opaque, ignoble, destinée aux ouvriers de l'histoire) et abstraction du texte (transparente, noble, destinée aux lectores), il revient à la poétique de penser la littérature indissociablement de ce qui la médiatise, de ce qui l'incorpore à nos pratiques, de ce qui l'inscrit dans notre réalité ordinaire. Elle est en ce cas aussi concernée quand elle prétend se lancer dans une quête psychologique ou herméneutique de type fondationnaliste - c'est-à-dire qui aspire à toucher un fond qui lui donnerait le dernier mot. Parce qu'elles ne peuvent être définies isolément les unes des autres, nos pratiques (littéraires) s'expliquent entre elles, et ne requièrent aucun fondement qui les transcenderait. Toute quête fondationnaliste d'une explication est une entreprise congénitalement minée, et le recours à une esthétique ou à des principes herméneutiques, est voué à l'échec, parce que tautologique et infalsifiable. Le développement des pratiques collagistes et des littératures numériques nous rappelle plutôt à la nécessité de congédier la psychologie de l'auteur individualisable et à le reconcevoir comme une interface fluctuant entre des réseaux (Weissberg, 2002) et amenée non plus à manipuler de grandes Idées, mais à bricoler avec ruse des fragments discursifs environnants, au sein de dispositifs (Quintyn, 2007).

En ce sens, on peut reconnaître la vanité de la recherche d'explications inexorablement circulaires, pour se contenter plutôt de décrire correctement cette totalité anthropologique - un jeu de langage adossé à une forme de vie ${ }^{(12)}$. La donne méthodologique s'en trouve changée : la critique littéraire serait invitée à ne produire que des paraphrases susceptibles de maximiser l'intelligibilité et l'efficacité du texte, et la poétique à enrichir notre arsenal catégoriel en vue d'une reconnaissance élargie des textes et d'un «lire-comme » plus ouvert ${ }^{(13)}$.

(12) Bien évidemment, la prétention à la description ne peut faire l'économie d'objections majeures : celle d'une impossible neutralité axiologique des catégories mobilisées pour mener la description, ainsi que celle d'une impossible distinction entre descriptif et normatif, entre fait et valeur (voir Putnam, 2002). Cette méfiance envers un idéal descriptiviste qui se prévaudrait d'une absolue innocuité est bien évidemment nécessaire (voir Schaeffer, 2011, chap. 4 et 5). Il n'en reste pas moins que l'ambition d'une description des pratiques (littéraires et a fortiori sociales) n'esquive pas la délicate question de l'inadéquation de nos catégories et participe d'un déflationnisme prudent à l'égard de nos prétentions ethnocentristes à l'explication.

(13) Dans les Recherches philosophiques (II, XI), Wittgenstein pose la notion de « voir-comme», en vertu de laquelle l'expérience de perception visuelle est indissociable d'un usage de catégories conceptuelles qui permettent la reconnaissance de tel ou tel objet comme appartenant à telle ou telle espèce. L'exemple célèbre du lapin-canard de Jastrow permettait à Wittgenstein d'affirmer que la vision était d'emblée un voir-comme (je le vois comme un lapin ou je le vois comme un canard, et ce de manière disjointe et non simultanée). On peut étendre cette remarque à l'expérience de lecture. On peut 


\section{Formes de vie et théories de l'action littéraire}

Restent à déterminer alors les contours pour le moins flottants d'une forme de vie littéraire. Que l'on nous permette à cet effet de tisser une analogie entre études littéraires et les Sciences Studies. Loin d'hypostasier son objet dans quelque ciel éthéré d'idéalités, le sociologue des sciences Harry Collins (1985) considère la science comme une " forme de vie », c'est-à-dire comme un ensemble de formules, de propositions, de savoirs et de théories qui ne serait que la partie émergée d'un iceberg fait principalement de savoir-faire, de connaissances tacites, de gestes, de routines et de techniques partagées au sein de communautés savantes, qui requièrent de la part de celui qui l'analyse une immersion ethnographique. Ce geste d'importation théorique lui permet de congédier une conception algorithmique et desséchée de la science, en vertu de laquelle d'une part la nature parlerait à travers les protocoles scientifiques une langue audible au chercheur et pour laquelle d'autre part les pratiques scientifiques seraient réductibles à une configuration finie de règles. La convocation de formes de vie littéraires devrait produire un effet heuristique similaire : pas plus que la science n'améliore notre compréhension du monde par l'entremise magique de ses formules et ses lois, la littérature ne s'appréhende pas dans une immédiateté textuelle abstraite et idéaliste. On a ainsi tort de réduire la littérature à de simples contenus logiques, ou de concevoir l'articulation littérature / vie à un niveau propositionnel, par exemple sur les modes de la magie, de la contamination ou de l'hypnose ${ }^{(14)}$. La littérature configure concrètement notre vie, certes par les postures et les gestes routiniers qu'elle convoque, mais encore et surtout par les techniques d'institution (ou de destitution) du sens et d'accords (ou de désaccords) qu'elle véhicule.

Décrire un jeu de langage littéraire reviendrait à concevoir la littérature comme une tactique destinée à donner toutes sortes de coups dans nos formes de vie. Il importe donc de braquer l'objectif sur des pratiques d'écriture dans leur mise en œuvre performative. Jean Bazin insiste à raison sur les effets mêmes de l'acte d'écriture historique, affirmant que tout « récit d'un événement est toujours lui-même un événement, dont le récit généralement ne parle pas » (2008, p. 289). L'écriture, irréductible à une source transparente, se laisse voir comme une production qui, non contente de dire le social, le produisent aussi, en se chargeant de valeurs spécifiques à la culture où elle émerge. L'écrit enregistre et consigne les actions antérieures, tout autant qu'il performe, en son aval, par sa mise en mots du réel, des morphologies sociales nouvelles. Alors qu'au sortir des révolutions du XIX ${ }^{\mathrm{e}}$ siècle, Tocqueville dressait «le tableau si vaste, si nouveau, si confus » d'une société " encore à moitié engagé[e] sous les débris du monde qui tombe » (Démocratie en Amérique, t. II, 4 ' partie, Chap. VIII), l'explosion du genre romanesque (de Balzac, d'Eugène Sue ou d'Alexandre Dumas), s'affairait à accroître la lisibilité de la société post-révolutionnaire en fournissant des ressources « descriptives et décryptives ", au point que la littérature panoramique des descriptions de Paris et les enquêtes sociales sur les classes dangereuses partageaient avec le roman une " manière commune de mettre en texte le monde social» (Lyon-Caen, 2003, p. 621). Si la littérature constitue bien, par l'instrument de l'écrit, un générateur d'interactions et de médiations sociales, ce que l'écrit donne à voir ne saurait exister sans la visibilité qu'il instaure et programme. Cela nécessite en ce cas de se doter d'une théorie de l'action historicisée pour

lire un texte comme de la poésie ou comme une inscription graphique ordinaire, comme un roman ou comme un journal intime. Pensons à l'expérience pédagogique bien connue de Stanley Fish, qui a soumis une bibliographie et la fait passer pour un poème mystique auprès de ses étudiants. Voir à cet égard Jocelyn Benoist (2006) et Baroni et Macé (2007, pp. 155-258).

(14) Voir à cet égard les critiques éclairantes de Hanna (2010, pp. 87-88). 
inspecter les rapports que chaque écrit entretient avec le monde et les possibilités d'intervention qu'il y favorise (Anheim et Chastang, 2009, p. 8).

Prenons l'exemple de l'hagiographie médiévale, qui a tant fait l'objet des investigations de la folkloristique, et qu' on a souvent confiné à une archive conservatrice au double sens du terme. Productrice d'une memoria communautaire et à ce titre dépositaire d'enjeux identitaires (à l'échelle d'une abbaye, d'une cité voire d'une nation en devenir) (Canetti, 1999), l'écriture du sacré qui s'y joue autorise et formalise en son aval des formes d'intervention nouvelles dans le monde. À cet égard les legendae novae, promues par l'ordre dominicain dans la seconde moitié du XIII siècle, et au premier rang desquelles la Légende dorée de Jacques de Voragine, compilent des vies de saints au sein de recueils calendaires et fournissent un stock d'arguments, d'exemples, d'images et des modèles comportementaux aux prédicateurs chargés d'édifier les fidèles. Ce sont autant d'instruments destinés à servir les ordres mendiants, bras armés d'une papauté en reconquête au sortir des croisades. Un tel livre participe à inscrire le lecteur dans une histoire téléologique du salut où toute action se conforme et se configure à l'événement de la Passion ${ }^{(15)}$. La légende et la parole du prédicateur qui en tire ses ressources nourrissent l'examen des multiples distorsions qui se glissent dans cette conformation et en favorisent l'extorsion de l'aveu. Elles fonctionnent à ce titre comme des opérateurs de subjectivation : s'affirmant comme les tuteurs d'une parole biographique et pénitentielle qu' elles font advenir par la confession, elles assujettissent le fidèle et tracent tout en même temps le cadre d'une émancipation par le salut. Un livre comme la Légende dorée se veut donc essentiellement le vecteur d'un gouvernement des âmes (Senellart, 1995) directement exercé sur un fidèle hétéronome et infantilisé, tel une brebis toujours sous la menace de l'égarement. Cette hagiographie qui se développe en même temps que la confession auriculaire et la procédure inquisitoire peut ainsi demeurer longtemps obscure ou étrange, à qui ne la connecte à la théorie de l'action pastorale qu'elle promeut mais dont les contours restent à dessiner. André-Georges Haudricourt (1962) l'a rapidement qualifiée d' " action directe positive », en proposant d'articuler techniques de domestication animale et de culture des plantes d'une part et techniques de gouvernement d'autre part. Foucault (2004), au moment où affleure la question de la gouvernementalité, détaille les modalités de l'action du pasteur, comme une forme de ministère, qui veille, accompagne, soigne ses ouailles, se dévoue pour elles avec bienfaisance, mais aussi exerce un contact permanent et une tutelle ferme, choisit à leur place et demande en contrepartie une obéissance sans faille. Cette littérature pastorale devient ainsi un laboratoire de formes normatives d'administration qui, loin de se désenchanter, s'intensifieront avec la modernité (Napoli, 2009 ; Büttgen, 2007).

Si une anthropologie pragmatique de la littérature rend justice à cette capacité qu'a la littérature d'ouvrir notre champ d'action dans le monde et de configurer nos formes de vie, les modalités pragmatiques de la littérature varient en fonction des formes de vie qu'elles informent en retour. La situation ultra-contemporaine d'une société si globalisée qu'elle ne laisse jamais entrevoir sa propre totalité qu'à travers un récit mondial du complot sans sujet ni référent (Jameson, 2007), appelle par exemple des logiques d'intervention adaptées, que les poésies les plus récentes modélisent et mettent en œuvre pour une grande part. À la manière d'un William Burroughs concevant la poésie comme un virus électronique, la poésie se donne pour fin de se glisser insidieusement dans nos représentations et nos discours pour cultiver à leur égard un certain soupçon, pour les dénaturaliser ou tout bonnement les faire muter. On peut également adosser la poésie contemporaine française (Denis Roche, Olivier Cadiot, Anne-James Chaton, Nioques, etc.) soit à une logique d'action situationniste, soit à une logique d'action directe, au

(15) Sur la configuratio comprise comme synthèse des figures chez les dominicains de San Marco à Florence, voir Didi-Huberman, 1990. 
sens où l'entendait Émile Pouget : une manière pour le sujet de puiser dans ses ressources pour saboter et miner le système qui l'oppresse (Hanna, 2002, 2010; Pouget, 2010).

En somme, informée d'une théorie de l'action adéquate à son objet, une anthropologie de la littérature fait éclater les limites strictes des analyses traditionnelles qui faisaient trop facilement de l'auteur le seul acteur de la littérature frontalement opposé à un lecteur passif et moutonnier. En lieu et place de ces cadres d'analyse, l'action artistique mérite de se requalifier, à la lumière des pratiques structurant les communautés où elles s'inscrivent, comme une coopération (Menger, 1993) entre une pluralité d'acteurs amenés à s'accorder pour agir ensemble. La littérature mérite d'être considérée comme un objet processuel, inscrit dans des faisceaux d'intentionnalités et des réseaux d'action et de pratiques, qu'il retravaille de l'intérieur et où auteur et lecteurs prennent place parmi une diversité d'autres acteurs souvent insoupçonnés. Et c'est cet ensemble qui constitue l'unité d'analyse de l'anthropologue de la littérature. Ainsi on évacuerait avec un réductionnisme desséchant un grand nombre de dimensions de l'hagiographie si l'on se contentait de l'envisager au sein d'une relation auteur et lecteurs. Il convient d'une part de prendre en compte les spécificités de l'écriture hagiographique : l'auteur d'une Vita n'est bien souvent qu'un relais qui compile et assemble des témoignages issus de la tradition - que ce soit un exemplum ou la pièce d'un procès de canonisation -, et sur lesquels il n'intervient que par le biais d'une réécriture (par amplification, abréviation, interpolation ou excision $)^{(16)}$. L'acte purement graphique de copie de la vie s'il est impulsé par un commanditaire peut chercher un transfert d'usages et réaménager les réseaux qui lient tous les acteurs autour de lui. La réécriture hagiographique partage avec la translation de reliques le projet d'un transfert d'usages, en vue d'une légitimation politique, d'une affirmation identitaire, d'une appropriation territoriale. On ne saurait ainsi déconnecter les fonctions de l'écrit hagiographique des modes d'existence du saint compris comme une intentionnalité à prendre en compte (y compris sous la forme des reliques et des dispositifs cultuels destinés à le présentifier).

Toutefois, en vertu des principes de communication différée et d'indétermination de sa destination, un texte littéraire est potentiellement réinscriptible dans des formes de vie nouvelles. Disponible, décontextualisable, remobilisable (Macé, 2009), mais jamais vagabond, il est une arme contextuellement déterminante et génératrice de prises inédites sur le réel, de manières concurrentes à la doxa de configurer le social, de présenter les problèmes publics (Dewey, 2010 ; Hanna, 2010) ou de rendre visible des minorités invisibles, bref de travailler de l'intérieur nos accords fondamentaux dans le langage, c'est-à-dire dans nos formes de vie (Wittgenstein, 2004, § 241 ; Descombes, 2002 ; Chauviré, 2010, pp. 83-102).

\section{A-t-on besoin de définition(s) de la littérature ?}

Car au fait, à force de diluer la littérature dans le langage ordinaire, on rétorquera à juste titre : de quelle littérature parle-t-on ? De l'hagiographie médiévale à la poésie contemporaine, en passant par le roman-feuilleton du XIX ${ }^{\mathrm{e}}$ siècle, les écarts génériques et historiques sont considérables et légitimement la question peut se poser de savoir ce qui nous autorise à réunir ces formes d'écrits sous une même catégorie littéraire. Il est probable que l'hagiographie médiévale et la poésie contemporaine ne possèdent pas de propriétés communes ou du moins que les raisons qui nous les fassent chacune qualifier de littéraire ne se recoupent guère. Il importe donc de répondre à la pressante question du corpus et du « terrain » d'investigation d'un anthropologue de la littérature.

(16) Voir 1'ensemble des recherches de Monique Goullet et Martin Heinzelmann (2003, 2005, 2006) autour des réécritures hagiographiques. 
Mais ce dernier a-t-il pour autant à répondre à la demande fondationnaliste, à la demande d'un critère fondamental de littérarité ? Précisément, la démarche anthropologique réside $i c i$, dans ce questionnement sur la possibilité ou l'impossibilité d'une identité littéraire clairement stabilisée. Si l'on s'accorde pour reconnaître que l'anthropologie est la discipline la mieux armée pour lever avec charité les facteurs d'incompréhension et les apparences d'irrationalité, alors une anthropologie de la littérature serait singulièrement la première à reconnaître les usages ambigus du mot « littérature ». Une définition de la littérature tend bien souvent à formuler le programme d'une œuvre d'art réussie (Schaeffer, 1992, pp. 360-361), de sorte que l'usage du mot est plus évaluatif que descriptif : il permet de décerner un certain degré d'excellence. Une anthropologie de la littérature serait la plus à même de mesurer les effets disqualifiants de la constitution d'un corpus dit littéraire, qui exclut en traçant la ligne de démarcation qui le distingue. Se donner avant toute chose une définition de la littérature avant de commencer une entreprise anthropologique à l'endroit de textes exotiques nous placerait dans une situation bien étrange, pour ne pas dire auto-mutilante. Nous serions dans une situation similaire à celle d'un ethnographe qui aurait méthodiquement et explicitement déjà statué sur son sens de l'humanité, avant même de commencer son enquête de terrain. Il serait ainsi pour le moins imprudent de disqualifier l'hagiographie médiévale sous prétexte qu'elle reste imprégnée de religiosité, qu'elle soit l'œuvre de compilateurs sans originalité, qu'elle prétende échapper à l'affabulation...

Ainsi, on pourrait suivre et adapter à notre propos la thèse de Morris Weitz (1988, p. 34) selon laquelle le concept d'art est résolument ouvert :

En ce qui concerne le concept d'art, ses conditions d'application ne peuvent jamais être énumérés exhaustivement puisque de nouveaux cas peuvent toujours être envisagés ou crées par des artistes, ou même par la nature, qui réclameraient une décision de la part de quelqu'un, afin d'étendre ou de clore l'ancien concept, ou d'en inventer un nouveau (par exemple « ce n'est pas une sculpture, c'est un mobile»).

À ceux qui, comme M. Weitz, voient dans l'art un concept expansif et imprévisible, et se privent de le définir, faute de pouvoir en énumérer tous les critères, le philosophe Noël Carroll répond que « cela n'a aucun sens de dire des œuvres d'art déjà faites qu'elles doivent être ouvertes à la permanente possibilité de changement et d'innovation. [...] Une définition de la science n'interdirait pas une découverte innovante, inattendue et sans préalable » (cité par Pouivet, 2001, p. 148). Il n'y a effectivement aucune implication logique entre l'absence de fondement philosophique des pratiques artistiques et l'impossibilité de définir ou d'identifier une œuvre d'art. Si poser une définition ne doit pas nous dispenser d'imaginer et d'innover, une définition ne clôt pas les évolutions contingentes de la littérature. Reste qu'une œuvre d'art déjà faite est ouverte à la possibilité de changement, d'innovation et de requalification rétroactive (Escola, 2005). Précisément : une œuvre d'art déjà constituée, du fait même de l'histoire de sa circulation, de sa diffusion qui l'ouvre à divers contextes d'utilisation échappe à tout contrôle et s'expose à diverses appropriations, canonisations ou déclassements (il n'est qu'à penser aux lettres de Madame de Sévigné ou à la poésie d'Anacréon étudiée par Florence Dupont (1994)).

La question est en ce sens moins celle - trop surplombante - de la définition, que celle de l'identification des œuvres littéraires. Cette position non essentialiste serait donc aussi disposée à ouvrir et étendre le sens de la littérature, à minorer certains piliers définitoires comme ceux de l'auctorialité, de la propriété, de l'originalité, de la créativité $^{(17)}$. Il s'agirait d'explorer les contrepoints et les contre-pieds faits à notre propre

(17) On peut renvoyer aux réflexions menées sur le terrain musical par Guillebaud, Mallet et Stoichita (2010). 
sens de la littérature. Si la question des modalités pratiques de l'identification des œuvres littéraires recouvre celle de la définition de la littérature, nos usages de la littérature suffisent à la définir :

Que l'art soit sans qualités, cela ne veut pas seulement dire que la possibilité en excède toujours quelque essence ou quelque origine que ce soit ; plus profondément, peutêtre, l'usage - en entendant par là ce qui ne saurait être détaché ni pensé indépendamment de ce que nous faisons - me semble en être la règle, l'erreur majeure consistant précisément à imaginer une règle antérieure à toutes ses applications, et qui en serait ainsi indépendante. (Cometti, 1999, p. 54)

Nous n'avons pas besoin d'une critériologie robuste du mot « littérature » pour mener à bien une anthropologie de la littérature : les définitions qui affleurent aujourd'hui dans nos corpus littéraires, et les usages sur lesquels elles reposent, suffisent - dans leur insuffisance - à lancer des opérations de décentrement comparatiste. Elles suffisent, parce que ce serait présumer de nos forces et du somme toute modeste recul historique que nous permet notre situation contemporaine à l'égard de textes candidats à la qualification littéraire.

Si nous reconnaissons, par ce geste non essentialiste, le caractère institutionnel et hétéronome de nos qualifications littéraires, nous n'en sommes pas moins à même de penser nos qualifications comme des actes politiques destinés à étendre nos usages de la littérature. Elles délivrent un surcroît, possiblement réparateur ou compensatoire, de représentations, une tribune alternative aux existences sous-estimées, minorées, marginales ou infâmes. De là plusieurs ensembles de textes s'ouvrent à nous : autant dans les franges de l'histoire littéraire, qu'elles soient chronologiques (médiévales et antiques) et culturelles (post-coloniales), qu'à travers toutes les formes de contiguïté entre écritures ordinaires et écritures littéraires ${ }^{(18)}$; autant les croisements transesthétiques (entre performance, musique, improvisation, dispositif, ready-made), que les métissages discursifs à l'œuvre dans les collages, dans les compilations, dans le sampling, qu'ils soient soutenus par d'anciens ou de nouveaux media technologiques. Et si nous n'aurions pas fini, il est vrai, d'apprendre à lire, c'est qu'une tâche assez noble incombe à l'anthropologue de la littérature : celui d'accroître la visibilité et la lisibilité des choses, des œuvres et du monde.

\section{Conclusions}

Si notre définition de l'art et de la littérature ne voyage guère hors des frontières géographiques ou historiques où une modernité restreinte se complait et requiert d'être corrigée par une anthropologie de la littérature, c'est qu'à de trop rares moments, la poétique a fait sienne la question politique du pouvoir du texte sur le contexte ${ }^{(19)}$. Le problème qui nous occupe prend racine et se réduit le plus souvent à un dialogue de sourds entre deux enfermements : l'enfermement textualiste dans une autotélie de l'œuvre et l'enfermement sociologique dans des déterminations sociales assimilées à des contraintes qui étouffent la liberté créatrice (Lahire, 2010). Ce blocage, loin d'être une alternative crédible, introduit une discontinuité frontale et ruineuse entre texte et con-

(18) Benoît Grévin (2008) montre par exemple combien les formes et les procédés de la diplomatique médiévale structurés par les artes dictaminis et les summe dictaminis dès le XIII ${ }^{\mathrm{e}}$ siècle ont forgé à la fois le langage de l'Etat moderne et fourni un arsenal discursif dont les premières élites humanistes $\mathrm{du} \mathrm{XV}^{\mathrm{e}}$ siècle ont fait largement leur miel.

(19) Soulignons avec Foucault (2001) la grammaire factitive du pouvoir, compris comme «action sur des actions ». 
texte, qu'on a tenté à de nombreuses reprises d'atténuer ou de tempérer. Les sens du « contexte » varient avec les types de continuité qu'on entend alors rétablir avec le texte : un historien de la lecture y voit les formes concrètes et matérielles du texte et les gestes routiniers impliqués par lui ; un sociologue de la littérature y verra plutôt un champ travaillé par des forces majoritaires et minoritaires, dominantes et dominées qui définissent un espace des possibles de la création; la sociocritique, quant à elle, comprenant le contexte comme un ensemble plus ou moins vaste de données discursives doxiques, se donne pour mission d'explorer les textes dans leur capacité d'ingestion et de retraitement de ces dernières.

Nous proposons, pour renouveler en partie la pensée des rapports entre texte et contexte, d'inverser ce rapport : le texte n'est pas circonscrit par le contexte, il redessine et refaçonne des contextes nouveaux. Le problème à résoudre et qui suppose de construire une anthropologie de la littérature est exactement celui que se pose Alfred Gell (2009) à l'égard des objets non occidentaux dits artistiques. Dès lors que l'on se rend compte que le concept d'art et l'approche esthétique qui lui est indexée sont inadéquats à leur endroit, l'élargissement nécessaire de perspectives conduit à considérer les objets à travers le pouvoir qu'ils exercent : leur agency. Voilà qui doit accroître notre attention aux effets que la littérature, comprise comme un ensemble divers et historicisé de jeux de langage, exerce sur notre vie.

La notion de forme de vie, élaborée par la seconde philosophie wittgensteinienne, nous semble de ce point de vue pertinente et toute désignée, en ce qu'elle désamorce un certain nombre de sophismes et de mythologies qui gangrènent les études littéraires : mythes d'une intériorité à exprimer, d'une signification cachée à exhumer, de la règle qui dérive vers une logicisation excessive du texte, mythe enfin d'une possible adéquation au réel (Chauviré, 2010, pp. 93-94)... La « forme de vie », dont nous proposons l'usage ici, ne se réduit pas à une nouvelle terminologie audacieuse qualifiant la contextualisation restreinte des conditions de production ou de réception qui président à la bonne compréhension de l'œuvre littéraire ; elle désigne ce que ne cesse d'informer et d'innerver, au niveau de nos pratiques ordinaires, la littérature. La littérature s'utilise en somme, et elle s'utilise au sein des autres usages qui font notre quotidien. Si l'on entend par usages ces « procédés que les individus mettent régulièrement en œuvre pour se servir des critères de jugement - impersonnels et publics - qui leur permettent d'identifier 'ce qui se passe' autour d'eux et de parvenir à faire converger ces identifications lorsqu'ils agissent »(Ogien, 2007, p. 13), alors la littérature se trouve richement dotée de pouvoirs à consolider ou déstabiliser, à réformer ou déformer, à clarifier ou parasiter ces usages et les formes de vie que ces acteurs tissent et voient se tisser entre eux. 


\section{Bibliographie}

Anheim, Étienne et Chastang, Pierre (2009) : Médiévales, n 56, « Pratiques de l'écrit », printemps.

BARONI, Raphaël et MACÉ, Marielle (2007) : Le savoir des genres, Rennes, PUR.

BAZIN, Jean (2008) : Des clous dans la Joconde, Toulouse, Anacharsis.

BENOIST, Jocelyn (2006) : «Voir comme quoi ?», Lire les Recherches Philosophiques de Wittgenstein, Paris, Vrin, pp. 237-253.

BESSIÈRE, Jean (2002) : Quel statut pour la littérature?, PUF, L'interrogation philosophique.

BLOOR, David (2001) : «Wittgenstein and the priority of practice», The Practice Turn in Contemporary Theory, T. R. Schatzki et al. dir., Londres/New York,k Routledge, pp. 104-114.

BOURDIEU, Pierre (1998) : Les règles de l'art. Genèse et structure du champ littéraire, Paris, Seuil.

BOUVERESSE, Jacques (2000) : «L'animal cérémoniel : Wittgenstein et l'anthropologie », Essais I. Wittgenstein, la modernité, le progrès et le déclin, Marseille, Agone, pp. 139-192.

- (2008) : La connaissance de l'écrivain. Sur la littérature, la vérité \& la vie, Marseille, Agone.

BÜtTGEN, Philippe (2007) : «Théologie politique et pouvoir pastoral », Annales HSS, $\mathrm{n}^{\circ} 5$, pp. 1129-1154.

BRANDOM, Robert (2009): L'articulation des raisons. Introduction à l'inférentialisme, trad. Claudine Tiercelin et Jean-Pierre Cometti, Paris, Cerf, Passages.

CANETTI, Luigi (1999) : L'invenzione della memoria, il culto e l'immagine di Domenico nella storia dei primi frati Predicatori, Firenze, SISMEL, Biblioteca di Medioevo Latino, 19.

CAVELL, Stanley (1991): Une nouvelle Amérique encore inapprochable. De Wittgenstein à Emerson, trad. Sandra Laugier-Rabaté, Combas, Éd. de l'Éclat.

- (1996) : Les voix de la raison. Wittgenstein, le scepticisme, la moralité et la tragédie, trad. Sandra Laugier et Nicole Balso, Paris, Seuil.

CERQUigLINI, Bernard (1989) : Éloge de la variante. Histoire critique de la philologie, Paris, Seuil, Des Travaux.

Chastang, Pierre (2008) : «L'archéologie du texte médiéval. Autour de travaux récents sur l'écrit au Moyen Âge », Annales HSS, n², pp. 245-269.

COLLINS, Harry (1985) : Changing Order : Replication and Induction in Scientific Practice, Londres / Beverly Hills / New Delhi, Sage Publications.

CoMETTI, Jean-Pierre (1999) : L'art sans qualités, Tours, Farrago.

- (2001) : Philosopher avec Wittgenstein, Tours, Farrago.

- (2009): La force d'un malentendu. Essais sur l'art et la philosophie de l'art, Paris, Questions théoriques, coll. Saggio Casino, 2009.

- (2010) : Qu'est-ce que le pragmatisme?, Paris, Gallimard, Folio Essais.

CHAUVIRÉ, Christiane (2004) : Le moment anthropologique de Wittgenstein, Paris, Kimé.

- (2010): Wittgenstein en héritage. Philosophie de l'esprit, Epistémologie, Pragmatisme, Paris, Kimé.

DAS Veena (1998) : «Wittgenstein and anthropology », Annual Review of Anthropology, $\mathrm{n}^{\circ} 27$, pp. 171-195.

DAVIDSON, Donald (1993) : Enquêtes sur la vérité et l'interprétation, trad. Pascal Engel, Nîmes, Jacqueline Chambon.

Descombes, Vincent (2002) : «L'idée de sens commun », Philosophia Scientiae, vol. 6/2, L'usage anthropologique du principe de charité, Isabelle Delpla dir., Paris, Kimé, pp. 147-161. 
— (2004) : Le complément de sujet. Enquête sur le fait d'agir de soi-même, Paris, Gallimard.

DEWEY, John (2010a) : L'art comme expérience, trad. J.-P. Canetti et al., Paris, Gallimard, Folio Essais.

— (2010b) : Le Public et ses problèmes, trad. J. Zask, Paris, Gallimard, Folio Essais.

Didi-Huberman, Georges (1990) : Fra Angelico. Dissemblance et figuration, Paris, Champs Flammarion.

DUPONT, Florence (1994) : L'invention de la littérature. De l'ivresse grecque au texte latin, Paris, La Découverte/Poche.

ESCOLA, Marc (2005) : «Des possibles rapports de la poétique et de l'histoire littéraire », dans « Théorie et histoire littéraire », Fabula LHT (Littérature, histoire, théorie), $\mathrm{n}^{\circ} 0$, [en ligne] [URL : http://www.fabula.org/ht/0/Escola.html]

Foster, Hal (2008) : Design et Crime, trad. C. Jaquet, Paris, Les Prairies Ordinaires.

FOUCAULT, Michel (2001) : «Le sujet et le pouvoir », Dits et Ecrits, t. II, 1976-1988, Gallimard, Quarto, pp. 1041-1062.

- (2004) : Sécurité, territoire, population. Cours au Collège de France, $1977-$ 1978, éd. établie par Michel Senellart, sous la dir. de François Ewald et Alessandro Fontana, Paris, Gallimard, Seuil.

GELL, Alfred (2009) : L'art et ses agents. Une théorie anthropologique, trad. D. et S. Renaut, Dijon, Les presses du réel, Fabula.

GOUllet, Monique (2003) : «Une typologie des réécritures peut-elle éclairer la nature du discours hagiographique ? », Hagiographica, 10, pp. 109-122.

Goullet, Monique et HeINZELMANN, Martin (dir.) (2003) : La réécriture hagiographique dans l'Occident médiéval. Transformations formelles et idéologiques, Paris, Thornbecke, Beihefte der Francia, p. 58.

- (2005): Écriture et réécriture hagiographiques. Essai sur les réécritures de Vies de saints dans l'Occident latin médiéval (VIIIe-XIIIe siècle), Turnhout, Brepols. - (2006) : Miracles, vies et réécritures dans l'Occident médiéval, Paris, Thornbecke, Beihefte der Francia 65.

GRÉVIN, Benoît (2008) : Rhétorique du pouvoir médiéval : les Lettres de Pierre de la Vigne et la formation du langage politique européen, XIIIe-XVe siècle, École française de Rome, BEFAR.

Guillebaud, Christine, MALlet, Julien et Stoichita, Victor A. (2010) : « La musique n'a pas d'auteur. Ethnographies du copyright », Gradhiva, n 12.

Hanna, Christophe (2002) : Poésie Action Directe, Romainville, Éd. Al Dante. - (2010) : Nos dispositifs poétiques, Paris, Questions théoriques, Forbidden Beach.

HAUDRICOURT, André-Georges (1962) : « Domestication des animaux, culture des plantes et traitement d'autrui », L'Homme, vol. 2, n 1, pp. 40-50.

JAMESON, Frederic (2007) : La totalité comme complot, trad. N. Vieillescages, Paris, Les prairies ordinaires.

JOAS, Hans (1999) : La créativité de l'agir, trad. P. Rusch, Paris, Cerf.

KALIFA, Dominique (2009) : «L'imprimé, le texte et l'historien : vieilles questions, nouvelles réponses? ", Romantisme, $\mathrm{n}^{\circ} 143 / 1$, pp. 93-99.

LACOUE-LABARTHE, Philippe et NANCY, Jean-Luc (1979) : L'absolu littéraire. Théorie de la littérature du romantisme allemand, Paris, Le Seuil (Poétique).

LAHIRE, Bernard, Franz Kafka. Éléments pour une théorie de la création littéraire, Paris, La Découverte, Textes à l'appui/Laboratoire des sciences sociales.

LARA, Philippe de (2005): Le rite et la raison. Wittgenstein anthropologue, Paris, Ellipses.

LATOUR, Bruno et WOOLGAR, Steve (1988) : La vie de laboratoire. La production des faits scientifiques, Paris, La Découverte, Sciences et société. 
LAUGIER, Sandra (2009) : Wittgenstein. Les sens de l'usage, Paris, Vrin, Moments philosophiques.

LYON-CAEN, Judith (2003) : « Histoire littéraire et histoire de la lecture », Revue d'histoire littéraire de la France, ${ }^{\circ} 3$, pp. 613-623.

MACÉ, Marielle (2009) : «Disponibilités littéraires : la lecture comme usage », Littérature, $\mathrm{n}^{\circ} 155$, pp. 3-21.

Maingueneau, Dominique (2006) : Contre saint Proust ou la fin de la Littérature, Paris, Belin, 2006.

MARTIN, Jean-Pierre, dir. (2010) : Bourdieu et la littérature, Nantes, Cécile Defaut.

MARY, André (2000) : Le Bricolage africain des héros chrétiens, Paris, Cerf.

MENGER, Pierre-Michel (1993) : « Machines et novateurs. Le compositeur et l'innovation technologique ", Les objets dans l'action. De la maison au laboratoire, Bernard Conein et al.. dir., Raisons pratiques.

MORIZOT, Jacques (2008) : «Enjeu cognitif et/ou théorie cognitive », Ce que l'art nous apprend. Les valeurs cognitives dans les arts, Sandrine Darsel et Roger Pouivet dir., Rennes, PUR, Aesthetica.

NAPOLI, Paolo (2009) : «La visita pastoral : un laboratorio de la normatividad administrativa »,Procesos, inquisiciones, pruebas. Homenaje a Mario Sbriccoli, E. Conte et M. Madero dir., Manatial, Buenos Aires, pp. 225-250.

NuSSBAUM, Martha (2010): La connaissance de l'amour. Essais sur la philosophie et la littérature, trad. Solange Chavel, Paris, Cerf, Passages.

OGIEN, Albert (2007) : Les formes sociales de la pensée. La sociologie après Wittgenstein, Paris, Armand Collin.

OLIVIER DE SARDAN, Jean-Pierre (1998) : «Émique », L'Homme, vol. 38, pp. 151-166.

POUGET, Emile (2010) : L'action directe et autres écrits syndicalistes (1903-1910), textes présentés par Miguel Chueca, Marseille, Agone.

POUIVET, Roger (2001) : «La quasi-nature des œuvres d'art», Sats Nordic Journal of Philosophy, vol. 2, n², Philosophia Press.

PUTNAM, Hilary (2004) : Fait/Valeur : la fin d'un dogme, et autres essais, trad. Marjorie Caveribère, Paris, Éditions de l'Éclat.

QUINE, W. V. O. (1977) : Le mot et la chose, trad. J. Dopp et P. Gochet, Flammarion.

QUINTYN, Olivier (2007) : Dispositifs/Dislocations, Paris, Al Dante/Questions théoriques, Forbidden Beach.

SAHLInS, Marshall (1976): The Critique of Pratical Reason, Chicago University Press.

SALGUES, Camille (2008) : "Un nouveau Wittgenstein encore inapprochable. Le rôle et la place du philosophe dans l'anthropologie », L'Homme, Miroirs transatlantiques, n'187-188, pp. 201-222.

SCHAEFFER, Jean-Marie (1992) : L'art de l'âge moderne. L'esthétique et la philosophie de l'art du XVIIIe siècle à nos jours, Paris, Gallimard.

— (2011) : Petite Écologie des Études Littéraires. Pourquoi et comment étudier la littérature?, Paris, Éditions Thierry Marchaisse.

SENELlaRT, Michel (1995): Les arts de gouverner. Du regimen médiéval au concept de gouvernement, Paris, Seuil, Des travaux.

VERnANT, Jean-Pierre (1996) : Mythe et pensée chez les Grecs. Etudes de psychologie historique, Paris, La Découverte.

Viveiros DECASTRO, Eduardo (2009) : Métaphysiques cannibales, trad. O. Bonilla, Paris, PUF, MétaphysiqueS.

WeISSBERG, Jean-Louis (2002) : «Copier-coller n'est pas plagier », Copier, Voler. Les Plagiaires, Critique, $\mathrm{n}^{\circ}$ 663-664, pp. 713-724

WitTGenstein, Ludwig (1983) : Remarques sur les fondements des mathématiques, trad. M. A. Lescourret, Paris, Gallimard.

— (2004) : Recherches philosophiques, trad. F. Dastur et al. Paris, Gallimard. 